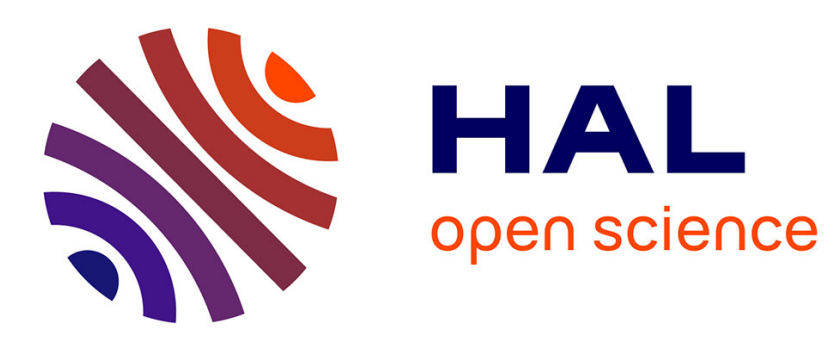

\title{
Relay-based hybrid control of minimal-order mechanical systems with applications
}

\author{
Andrea Bisoffi, Fulvio Forni, Mauro da Lio, Luca Zaccarian
}

\section{To cite this version:}

Andrea Bisoffi, Fulvio Forni, Mauro da Lio, Luca Zaccarian. Relay-based hybrid control of minimal-order mechanical systems with applications. Automatica, 2018, 97, pp.104-114. 10.1016/j.automatica.2018.07.033 . hal-01970879

HAL Id: hal-01970879

https://hal.laas.fr/hal-01970879

Submitted on 6 Jan 2019

HAL is a multi-disciplinary open access archive for the deposit and dissemination of scientific research documents, whether they are published or not. The documents may come from teaching and research institutions in France or abroad, or from public or private research centers.
L'archive ouverte pluridisciplinaire HAL, est destinée au dépôt et à la diffusion de documents scientifiques de niveau recherche, publiés ou non, émanant des établissements d'enseignement et de recherche français ou étrangers, des laboratoires publics ou privés. 


\title{
Relay-based hybrid control of minimal-order mechanical systems with applications *
}

\author{
Andrea Bisoffi ${ }^{\text {a }}$, Fulvio Forni ${ }^{\mathrm{b}}$, Mauro Da Lio $^{\mathrm{a}}$, Luca Zaccarian ${ }^{\mathrm{c}, \mathrm{a}}$ \\ ${ }^{a}$ Dipartimento di Ingegneria Industriale, University of Trento, Italy \\ ${ }^{\mathrm{b}}$ Department of Engineering, University of Cambridge, United Kingdom
}

${ }^{\mathrm{c}}$ CNRS, LAAS, 7 avenue du Colonel Roche, F-31400 Toulouse, France and Université de Toulouse, 7 avenue du Colonel Roche, 31077 Toulouse cedex 4, France

\begin{abstract}
This work explores the potential of relay-based control on a one-degree-of-freedom nonlinear mechanical system, in the contexts of both sustaining and damping oscillations. For both cases we state our main results building upon a simple reset formulation (relay feedback) and providing intuitive basic equations from classical mechanics. With a more rigorous description following a hybrid system formalism, we establish then the global asymptotic stability of the corresponding (compact-set) attractors through hybrid Lyapunov tools. The aspects of sustaining and damping oscillation are seen as complementary, because they reduce to a suitable mirroring of the reset surface. Finally, we discuss two applications of our results to the case of a hopping mass and an automotive suspension.
\end{abstract}

Key words: Nonlinear control systems, nonlinear systems, relay control, reset actions, discontinuous control, forced oscillations, periodic motion, limit cycles, global stability, asymptotic stability, Lyapunov stability, stability analysis, Lyapunov methods, mechanical systems

\section{Introduction}

Linear plants in feedback with nonlinear controllers in the form of relays with hysteresis have been studied since the 1950's [20,45] (and also [44]) thanks to the favorable features [2] of the power amplifiers implementing the relays. More generally, at that time it has been recognized with the pioneering work of [12] that reset actions in control systems may improve upon the potential of linear designs (this fact was rigorously proven only recently in [7]). Follow-up research on reset control comprises the introduction of the concept of First Order Reset Element (FORE) by Horowitz [24], whose use in control systems design in subsequent papers is well summarized in $[7,8,11]$ and references therein. In re-

\footnotetext{
* The material of this paper was partially presented at the 2016 European Control Conference. Research supported in part by CaRiTRo through grant PowerLyap. Corresponding author A. Bisoffi.

Email addresses: andrea.bisoffi@unitn.it (Andrea Bisoffi), f.forni@eng.cam.ac.uk (Fulvio Forni), mauro.dalio@unitn.it (Mauro Da Lio), zaccarian@laas.fr (Luca Zaccarian).
}

cent years, a revived interest in the field of FORE-based reset control emerged from the availability of new theoretical tools for the analysis of nonlinear hybrid systems $[17,18]$, which motivated more recent works well represented by $[3,13,40]$ and references therein.

Paralleling the above mentioned research strand, several works such as [1] (see also [29, §18.1.8]) have testified the potential of reset control for sustaining oscillations, which then extends quite naturally to the problem of generating hybrid limit cycles via hybrid feedback for legged locomotion $[27,28,37,48]$ and for the close setting of juggling systems [39]. The potential of applying hybrid dynamical systems techniques in the context of legged locomotion is pointed out in [19], and addressed in technical terms in [43]. More closely related to this work, the hopping mass is the first milestone in legged locomotion for robots, as witnessed by the impact of the seminal work [36] (see also the more recent [42] and references therein). On a parallel thread, it must be recognized that relay-based feedback has proven to be effective also in damping oscillations, which may find applications in neuroscience [33] but also in the context of semi-active suspensions in the automotive field, as one 
may appreciate from the survey works in [35, Section 4$]$ and [41, Chapter 6].

Motivated by the above observations, in this paper we apply a reset control paradigm to a nonlinear mechanical system in order to sustain oscillations and, in a complementary way, to damp them (in the linear case, one of our results relates to [1]). Furthermore, in the same spirit of $[15,16]$ (or [23] for a broader approach encompassing neurobiology and biomechanics), we use minimal order mechanical systems to provide a fundamental explanation to the phenomena of reset-sustained and reset-damped oscillations. Finally, our work is motivated by some newly devised actuators [28] for hopping locomotion $[37,48]$, whose very fast action resembles the introduction of a "kick" of energy to the mechanical system and can be modeled by a controller reset (an alternative approach close to the nature of this work would be [38]). Similar types of actuation are used in [6,27] and in variable impedance actuators [46].

In comparison to $[4,5,30-32]$, here we consider sustaining and damping oscillations (in a mechanical system) as complementary. The nature of the approach in [30-32] is close to our approach for the case of sustained oscillations, although we do not require any a priori knowledge about the existence of a hybrid periodic solution (as, for instance, in [30, Assumption 4.5, item 4)]). In [4,5], limit cycles are an undesired dynamics arising when stabilizing through linear resets the origin of a linear system; [5] relies on the matrix exponential for the Poincaré map, and [4] utilizes the approximate method of the describing function [25, Section 7.2]. Our underlying system is instead a nonlinear (one-degree-of-freedom) mechanical system; our approach is based on Poincaré map and Lyapunov analysis.

As main contributions, this work focuses on exploring relay-based laws to sustain and damp oscillations for one-degree-of-freedom nonlinear mechanical systems. The analysis is based on hybrid dynamical systems tools $[17,18]$, adapted in particular to the study of periodic orbits. The resulting framework is justified and illustrated through two relevant engineering applications. This work extends [10], whose problem setting originates from [26], by considering more general nonlinear mechanical systems: the study of sustained oscillations is more detailed and the study of reset-damped oscillations and applications is new. The paper is structured as follows. Sections 2 and 3 present reset-sustained and reset-damped oscillations, respectively. Sections 2.1 and 3.1 provide the main results of the paper and Sections 2.2 and 3.2 discuss technical details and proofs. Our proofs are based on $[17,18]$ and take inspiration from classical Poincaré analysis [21]. Applications are illustrated in Section 4 with a simple model of a hopping robot and with a (semi-active) suspension from the automotive field, respectively.

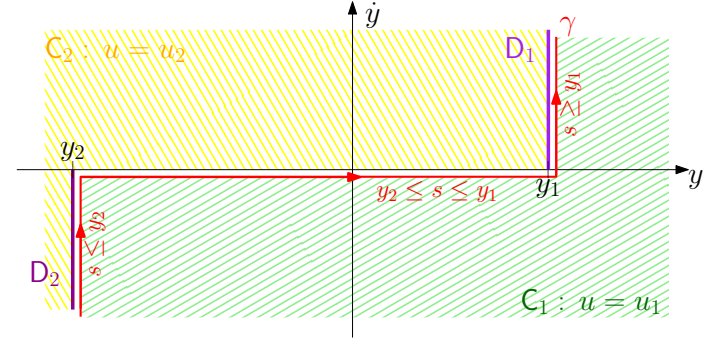

Figure 1. Curve $s \mapsto \gamma(s)$ in red and related sets $\mathrm{C}_{i}$ and $\mathrm{D}_{i}$.

Notation. The nonnegative reals and integers are denoted by $\mathbb{R}_{\geq 0}$ and $\mathbb{Z}_{\geq 0}$, respectively. The domain of a function $f$ is denoted by $\operatorname{dom} f$ and in the specific case of a solution $\phi$ to a hybrid dynamical system, dom $\phi$ denotes a hybrid time domain as in [18, Definition 2.3]. For a set $\mathrm{S}, \overline{\mathrm{S}}$ denotes its closure. The closed unit ball is denoted by $\mathbb{B}$.

\section{Reset-sustained oscillations}

\subsection{General theory}

Consider the one-degree-of-freedom nonlinear mechanical system

$$
m \ddot{y}+c(y, \dot{y}) \dot{y}+\frac{\partial U}{\partial y}(y, u)=0
$$

where $y$ is the position, $\dot{y}$ is the velocity, $\ddot{y}$ is the acceleration, $m$ is the mass, $c(y, \dot{y})$ is the nonlinear damping coefficient, and $U(y, u)$ is the nonlinear potential whose dependence on the position $y$ is modulated by $u$, which we use as a control input to the system. We show that system (1a) can be controlled into steady state oscillations by simple, piecewise constant, reset laws. Based on Figure 1, consider the curve

$$
\gamma(s):= \begin{cases}\left(y_{2}, s-y_{2}\right) & s \leq y_{2} \\ (s, 0) & y_{2} \leq s \leq y_{1} \\ \left(y_{1}, s-y_{1}\right) & s \geq y_{1}\end{cases}
$$

that divides the plane $(y, \dot{y})$ into the two regions

$$
\begin{aligned}
& \mathrm{C}_{1}:=\left\{(y, \dot{y}):\left(\dot{y}<0, y_{2}<y \leq y_{1}\right) \text { or } y>y_{1}\right\} \\
& \mathrm{C}_{2}:=\left\{(y, \dot{y}):\left(\dot{y}>0, y_{2} \leq y<y_{1}\right) \text { or } y<y_{2}\right\}
\end{aligned}
$$

where $y_{1}>y_{2}$ are two constant values. We pursue minimal actuation complexity, so we restrict ourselves to a binary control action $u$ depending on the state $(y, \dot{y})$ as

$$
u= \begin{cases}u_{1} & \text { if }(y, \dot{y}) \in \mathrm{C}_{1} \\ u_{2} & \text { if }(y, \dot{y}) \in \mathrm{C}_{2}\end{cases}
$$

where $u_{1}$ and $u_{2}$ are two constant values. At the same time, we also keep a minimal sensing complexity because 
the resets are triggered when the state $(y, \dot{y})$ is detected to cross the branches

$$
\begin{aligned}
& \mathrm{D}_{1}:=\left\{\gamma(s): s \geq y_{1}\right\} \\
& \mathrm{D}_{2}:=\left\{\gamma(s): s \leq y_{2}\right\} .
\end{aligned}
$$

Models similar to (1) can be found in $[26,27,48]$ wherein the use of such models is extensively motivated in the context of robotic applications. Indeed, we further discuss the practical relevance of this model in Section 4 . Solutions to (1) are well defined in view of their hybrid definition that we postpone to the next Section 2.2. We consider then the following standard concepts.

Definition 1 Orbit denotes the image of a solution to (1). A periodic solution is a solution $\psi$ defined for all nonnegative times for which there exists $T>0$ such that $t \in \operatorname{dom}(\psi)$ implies $t+T \in \operatorname{dom}(\psi)$ and, moreover,

$$
\psi(t)=\psi(t+T) \quad \forall t \geq 0 .
$$

$A$ periodic orbit is the image of a periodic solution, and is nontrivial if it comprises more than one point.

Based on this definition, we can characterize asymptotic stability for (1) according to the classical notions for nonlinear systems. The resets act as a "kick" of energy for the system, with oscillations arising from the balance between the energy introduced by the resets and the energy dissipated during the flow. The uniqueness of a periodic orbit that is globally asymptotically stable is guaranteed under mild conditions on the reset law and on the system nonlinearities.

Assumption 1 Given $y_{1}>y_{2}$, the function $(y, \dot{y}) \mapsto$ $c(y, \dot{y})$ is differentiable in each of the domains $\overline{\mathrm{C}}_{1}$ and $\overline{\mathrm{C}}_{2}$, and the functions $y \mapsto \frac{\partial U}{\partial y}\left(y, u_{1}\right)$ and $y \mapsto \frac{\partial U}{\partial y}\left(y, u_{2}\right)$ are locally Lipschitz in $\overline{\mathrm{C}}_{1}$ and $\overline{\mathrm{C}}_{2}$, respectively. Moreover, for some $c_{\epsilon}>0$,

$$
\begin{aligned}
& c(y, \dot{y}) \geq c_{\epsilon}>0 \text { for all }(y, \dot{y}) \\
& \left.\frac{\partial c}{\partial \dot{y}}(y, \dot{y}) \geq 0 \text { for all }(y, \dot{y}) \text { such that } \dot{y}>0\right\} \\
& \left.\frac{\partial c}{\partial \dot{y}}(y, \dot{y}) \leq 0 \text { for all }(y, \dot{y}) \text { such that } \dot{y}<0\right\} \\
& \left.\begin{array}{c}
\frac{\partial U}{\partial y}\left(y, u_{1}\right)>0 \text { for all } y \text { such that }(y, \dot{y}) \in \overline{\mathrm{C}}_{1} \\
\frac{\partial U}{\partial y}\left(y, u_{2}\right)<0 \text { for all } y \text { such that }(y, \dot{y}) \in \overline{\mathrm{C}}_{2} .
\end{array}\right\}
\end{aligned}
$$

Condition (2a) is trivially satisfied by any (nonideal) mechanical system. Conditions (2b) and (2c) restrict the nonlinearity of the damping coefficient and the potential to monotone functions in regions defined by the sign of $\dot{y}$ and in the regions $\bar{C}_{i}$, respectively. Condition (2b) includes the case of constant damping and, for instance, the case of a cubic damping that is considered in $[22,34]$, which illustrate the advantages of such a damping for vibration isolation (cf. Theorem 2 in Section 3.1). We have the following general result.

Theorem 1 For the mechanical system (1) satisfying Assumption 1, there exists a unique nontrivial periodic orbit that is globally asymptotically stable.

Remark 1 The stability result in Theorem 1 is proven in Section 2.2 using a hybrid representation whose regularity features enable the use of the fundamental properties established in [18]. These properties are instrumental in concluding global $\mathcal{K} \mathcal{L}$-asymptotic stability of the periodic orbit [18, Theorem 7.12] and robustness of this stability property to a fairly general class of perturbations that vanish on the periodic orbit [18, Theorem 7.21]. For more general perturbations, possibly arising from external disturbances or numerical approximations of the control signal, [18, Lemma 7.20] establishes a convenient notion of semiglobal practical asymptotic stability essentially comprising a graceful deterioration of the established stability properties as the size of the disturbance/uncertainty becomes larger. While we state this robustness in Proposition 1 in Section 2.2, we do not enter the details of its definition because the main focus of this paper is on nominal properties.

Remark 2 The result in Theorem 1 is connected to the classical contribution [1], where the input to a linear time-invariant single-input-single-output system is obtained through a feedback relay and necessary conditions are provided for the local stability of the resulting periodic solution, possibly in the presence of time delays on the input. The same setting but with an asymmetric relay has been investigated very recently in [9] due to its more beneficial features for PID autotuning in terms of persistent excitation. In the current work, the previous Theorem 1 provides sufficient conditions for global asymptotic stability of a fully nonlinear mechanical system for asymmetric oscillations, that is, $\left|u_{1}\right| \neq\left|u_{2}\right|$ and $\left|y_{1}\right| \neq\left|y_{2}\right|$.

Example 1 We illustrate Theorem 1 on a simple massspring-damper whose nonlinear stiffness is modulated by the input $u$ (compare with $[46, \S 3.3]$ ). We choose the parameters $m=1 \mathrm{~kg}, c=0.3 \mathrm{Ns} / \mathrm{m}, \frac{\partial U}{\partial y}(y, u)=$ $4(y-u)-\frac{5}{3}(y-u)^{3}+\frac{1}{5}(y-u)^{5}$. The potential gradient $\frac{\partial U}{\partial y}(y, 0)$ is the black dashed curve in the right part of Figure 2, which we design to have a local minimum that could represent the drop in the stress curve after a yield point for ductile materials. As for the reset law, fix $u_{2}=-u_{1}=0.75 \mathrm{~m}$ and $y_{1}=-y_{2}=0.75 \mathrm{~m}$, in a symmetric fashion. The control action $u$ translates horizontally $\frac{\partial U}{\partial y}(\cdot, u)$, so that $(2 \mathrm{c})$ is satisfied. The results are shown in Figure 2. On the left, orbits for different initial conditions are depicted together with the periodic orbit 

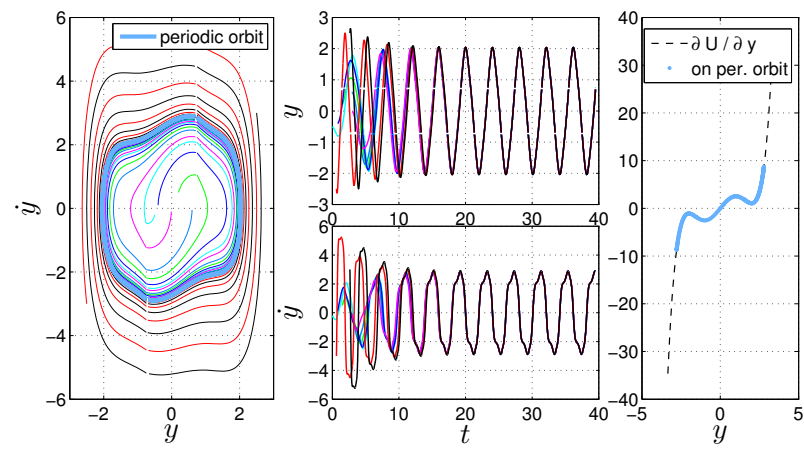

Figure 2. Example 1 for a nonlinear potential gradient. Left: periodic orbit. Center, top: position $y$. Center, bottom: velocity $\dot{y}$. Right: potential gradient curve (black), and portion explored when $y$ is on the periodic orbit (light blue).

they converge to. In the center, we plot the corresponding solutions $y$ and $\dot{y}$ for the same initial conditions. On the right, we add to the dashed curve $\frac{\partial U}{\partial y}(y, 0)$ (shown in black) the pairs $\left(y, \frac{\partial U}{\partial y}(y, 0)\right)$ (shown in light blue) such that $y$ lies on the periodic orbit depicted with the same color in the left part of the figure.

\subsection{Hybrid formulation and proof of Theorem 1}

In the previous section, solutions were parametrized only by the ordinary time $t$ as $\psi: \mathbb{R}_{>0} \rightarrow \mathbb{R}^{2}$, and the results involved the classical notions of asymptotic stability of compact sets (the unique periodic orbit in Theorem 1) for nonlinear continuous-time systems. Assume now that we track the number of resets $j$ undergone by the solutions, and that we make this additional parametrization explicit in the solution as $\hat{\psi}: \mathbb{R}_{>0} \times \mathbb{Z}_{>0} \rightarrow \mathbb{R}^{2}$. Consider also the control action $u$ as a state, which is constant whenever $(y, \dot{y})$ belongs to $C_{1}$ or $C_{2}$ and is updated at a reset.

These points lead seamlessly to a formulation according to the hybrid dynamical systems in [18]: continuous evolution of solutions is allowed in a flow set $\mathcal{C}$ according to a differential equation, discrete evolution (here, resets of $u$ ) is allowed in a jump set $\mathcal{D}$ according to a difference equation. Using the pictorial representation in Figure 3, we write then (1) as

$$
\begin{aligned}
& \left.\begin{array}{l}
m \ddot{y}+c(y, \dot{y}) \dot{y}+\frac{\partial U}{\partial y}(y, u)=0 \\
\dot{u}=0
\end{array}\right\} \quad(y, \dot{y}, u) \in \mathcal{C} \\
& y^{+}=y \\
& \left.\dot{y}^{+}=\dot{y}\right\} \quad(y, \dot{y}, u) \in \mathrm{D}_{i} \times\left\{u_{3-i}\right\}, i \in\{1,2\} \\
& u^{+}=u_{i} \\
& \mathcal{C}:=\mathcal{C}_{1} \cup \mathcal{C}_{2}:=\left(\bar{C}_{1} \times\left\{u_{1}\right\}\right) \cup\left(\overline{\mathrm{C}}_{2} \times\left\{u_{2}\right\}\right) \\
& \mathcal{D}:=\mathcal{D}_{1} \cup \mathcal{D}_{2}:=\left(\mathrm{D}_{1} \times\left\{u_{2}\right\}\right) \cup\left(\mathrm{D}_{2} \times\left\{u_{1}\right\}\right)
\end{aligned}
$$

where $\bar{C}_{1}$ and $\bar{C}_{2}$ denote the closures of $C_{1}$ and $C_{2}$ in (1c)$(1 d)$, and $D_{1}$ and $D_{2}$ were defined in (1f)-(1g). Thanks to

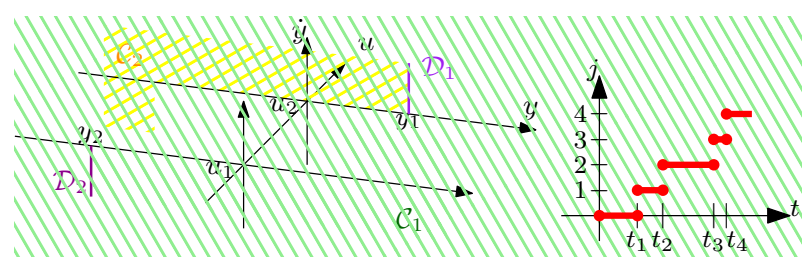

Figure 3. Left: hybrid system formulation (3) of system (1). Right: a typical hybrid time domain for (3) (see (6) for the definition of $\left.t_{1}, \ldots, t_{4}\right)$.

the shift from (1) to a hybrid system formulation in (3) and thanks to its regularity (in the sense of [18, Assumption 6.5]), we can establish in the proof of Proposition 1 that the periodic orbit is globally asymptotically stable straightforwardly from the characterization of the behaviour of solutions through the (hybrid) Poincaré map analyzed in Lemma 2.

The concept of solution for a hybrid dynamical system like (3) builds upon the notion of hybrid time domains [18, Definition 2.3], which involve the two time directions $t$ and $j$ and whose typical appearance for (3) is in Figure 3 (cf. Lemma 1). The solution concept for a hybrid dynamical system is detailed in [18, Definition 2.6]. Then, given a hybrid solution $\phi$ with hybrid time domain dom $\phi$, define the function that associates to each time $t$ the least index $j$ such that $(t, j) \in \operatorname{dom} \phi$ as

$$
\underline{j}(t):=\min _{(t, j) \in \operatorname{dom} \phi} j .
$$

Using (4), we can project a hybrid solution $(t, j) \mapsto$ $\phi(t, j)=(y(t, j), \dot{y}(t, j), u(t, j))$ onto the ordinary time direction as $t \mapsto \phi(t, \underline{j}(t))=: \bar{\phi}(t)=(\bar{y}(t), \overline{\dot{y}}(t), \bar{u}(t))$. Such $\bar{\phi}$ satisfies by construction (1e) and, given the signal $^{1} t \mapsto \bar{u}(t),(\bar{y}, \overline{\dot{y}})$ is a solution to the following reformulation of (1a)

$$
m \ddot{y}+c(y, \dot{y}) \dot{y}+\frac{\partial U}{\partial y}(y, \bar{u}(t))=0
$$

in the sense of Carathéodory as in [14, page 3]. Then, solutions to (3) are also solutions to (1), and since the former are well-defined, for the latter we were allowed to define the concepts in Definition 1, and characterize the stability properties in Theorem 1, as we did in Section 2.1. We can then parallel Definition 1 as follows.

Definition 2 Orbit denotes the image of a hybrid solution to (3). A hybrid periodic solution is a complete ${ }^{2}$ solution $\phi$ for which there exists a pair of nonnegative scalars $(T, J)$ with $T+J>0$, such that $(t, j) \in \operatorname{dom} \phi$

\footnotetext{
$\overline{1} \operatorname{By}(4), \bar{u}$ is left-continuous.

2 A solution is complete if its domain is unbounded [18, pages 28 and 30].
} 
implies $(t+T, j+J) \in \operatorname{dom} \phi$ and, moreover,

$$
\phi(t, j)=\phi(t+T, j+J), \quad \forall(t, j) \in \operatorname{dom} \phi .
$$

$A$ hybrid periodic orbit is the image of a hybrid periodic solution, and is nontrivial if it comprises more than one point.

First we establish in Lemma 1 the properties of maximal solutions [18, Definition 2.7] that we need for Lemma 2, among which uniqueness and completeness.

Lemma 1 For each initial condition, maximal solutions to (3) are unique, each flow interval of their hybrid time domain is bounded, they jump from $\mathcal{D}_{1}$ infinitely many times and they are complete.

Proof of Lemma 1. We divide the proof in three steps each proving a part of our statement, and we always refer to Figure 3.

(a) For each initial condition, maximal solutions to (3) are unique.

The flow and jump maps in (3a)-(3b) are locally Lipschitz single-valued functions by Assumption 1, and no flow is possible from the jump set because in $\mathcal{C} \cap \mathcal{D}_{1}$ $\left(\mathcal{C} \cap \mathcal{D}_{2}\right)$ the velocity $\dot{y}$ is positive (negative, respectively).

(b) For each initial condition, each flow interval of the hybrid time domain of the maximal solution to (3) is bounded.

Take any initial condition in $\mathcal{C}_{1}$. The maximal solution is bound to leave in finite time the set $\mathcal{C}_{1} \cap\{(y, \dot{y}, u): \dot{y} \geq 0\}$ because

$$
x \in \mathcal{C}_{1} \cap\{(y, \dot{y}, u): \dot{y} \geq 0\} \Longrightarrow \ddot{y}<0
$$

since $m \ddot{y}=-c(y, \dot{y}) \dot{y}-\frac{\partial U}{\partial y}\left(y, u_{1}\right)<0$ thanks to $(2 \mathrm{a})$ and (2c). Moreover, after crossing $\dot{y}=0, \dot{y}$ remains negative and bounded away from zero, so that $\mathcal{D}_{2}$ is reached in finite time. A parallel reasoning holds for any initial condition in $\mathcal{C}_{2}$. Then each flow interval is bounded.

(c) For each initial condition, the maximal solution to (3) jumps from $\mathcal{D}_{1}$ infinitely many times and it is complete. By the argument in the previous step (b), a maximal solution in $\mathcal{C}_{1}$ necessarily reaches $\mathcal{D}_{2}$ in finite time and the jump map in $(3 \mathrm{~b})$ guarantees that a solution in $\mathcal{D}_{2}$ necessarily reaches $\mathcal{C}_{2}$. The same argument guarantees then that a solution in $\mathcal{C}_{2}$ necessarily reaches $\mathcal{D}_{1}$ in finite time and the jump map guarantees that a solution in $\mathcal{D}_{1}$ reaches $\mathcal{C}_{1}$. We can then conclude that maximal solutions jump infinitely many times from $\mathcal{D}_{1}$, which implies completeness [18, Definition 2.5 and page 30$]$.

Thanks to Lemma 1, we can parametrize (maximal) solutions by their initial condition $x_{0}=\left(y^{0}, \dot{y}^{0}, u^{0}\right)$ as $(t, j) \mapsto \phi^{x_{0}}(t, j)=\left(\phi_{y}^{x_{0}}(t, j), \phi_{\dot{y}}^{x_{0}}(t, j), \phi_{u}^{x_{0}}(t, j)\right)$. In the following we consider extensively solutions with $x_{0}$ in $\mathcal{D}_{1}$. Moreover, given a hybrid solution $\phi^{x}$ with hybrid time domain dom $\phi^{x}$, define the function that associates with each jump index $j$ the least time $t$ such that $(t, j) \in$ $\operatorname{dom} \phi^{x}$ as

$$
t_{j}:=\min _{(t, j) \in \operatorname{dom} \phi^{x}} t
$$

corresponding then to the time when the jump index increases from $j-1$ to $j$. Based on the parametrization by the initial condition and on (6), we define for each $x \in \mathcal{D}_{1}$ the function $P: \mathcal{D}_{1} \rightarrow \mathcal{D}_{1}$ as

$$
P(x):=\phi^{x}\left(t_{3}, 2\right),
$$

which is well defined because of Lemma 1 and plays the role of a Poincaré map for the equation

$$
x^{+}=P(x), \quad x \in \mathcal{D}_{1} .
$$

By adapting the classical Poincaré analysis in [21] to the specific hybrid setting, the existence of a hybrid periodic orbit follows from the properties of $P$ that are presented in the next Lemma.

Lemma 2 The function $P: \mathcal{D}_{1} \rightarrow \mathcal{D}_{1}$ is continuous. There exists a unique equilibrium

$$
x^{\star}=P\left(x^{\star}\right),
$$

which is globally asymptotically stable for (8).

Proof of Lemma 2. We divide the proof into steps.

The function $P: \mathcal{D}_{1} \rightarrow \mathcal{D}_{1}$ is continuous.

Lemma 1 guarantees that for each initial condition (hence, for one in $\mathcal{D}_{1}$ ), solutions reach $\mathcal{D}_{1}$ in finite time. In particular, any solution reaches $\mathcal{D}_{1}$ after a first jump occurring at $t_{1}=t_{0}=0$, a flowing interval $\left[t_{1}, t_{2}\right]$, a second jump at $t_{2}$, and a second flowing interval $\left[t_{2}, t_{3}\right]$. Therefore, according to (7), the range of $P$ is indeed $\mathcal{D}_{1}$.

Since the sets $\mathcal{C}$ and $\mathcal{D}$ in (3c)-(3d) are closed and the flow and jump maps in (3a)-(3b) are continuous functions by Assumption 1, hybrid system (3) satisfies the so-called hybrid basic conditions [18, Assumption 6.5], which imply nominal well-posedness [18, Theorem 6.8] in the sense of $[18$, Definition 6.2]. Since the (unique, maximal) solution $\phi^{x_{0}}$ to (3) from $x_{0} \in \mathcal{D}_{1}$ is complete by Lemma 1, [18, Proposition 6.14] concludes that for every $\epsilon>0$ and $\tau \geq 0$ there exists $\delta>0$ such that for every $\phi^{x}$ (with $x \in \overline{\mathcal{D}}_{1}$ and $\left|x-x_{0}\right| \leq \delta$ ), $\phi^{x}$ and $\phi^{x_{0}}$ are $(\tau, \epsilon)$-close [18, Definition 5.23]. In particular, this definition implies that for $\tau$ selected as the sum of two jumps and the (finite) ordinary times needed by $\phi^{x_{0}}$ to traverse $\mathcal{C}_{1}$ and $\mathcal{C}_{2}$, there exists $s$ such that $(s, 2) \in \operatorname{dom} \phi^{x}$ and

$$
\begin{aligned}
& \left|\phi^{x}(s, 2)-P\left(x_{0}\right)\right|= \\
& \quad\left|\left(\phi_{y}^{x}(s, 2), \phi_{\dot{y}}^{x}(s, 2), u_{2}\right)-\left(y_{1}, P_{\dot{y}}\left(x_{0}\right), u_{2}\right)\right|<\epsilon
\end{aligned}
$$




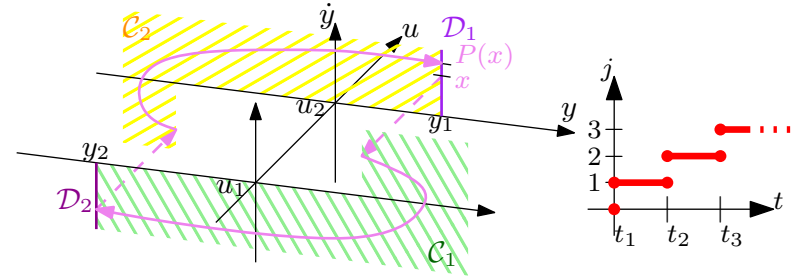

Figure 4. Left: the orbit of $\phi^{x}$ (violet). Right: its hybrid time domain.

where $P\left(x_{0}\right):=\phi^{x_{0}}\left(t_{3}, 2\right)$ by $(7)$ and $P_{\dot{y}}\left(x_{0}\right)$ is defined as the velocity component of $P\left(x_{0}\right): \tau$ is selected as above and $\epsilon$ will be selected later. To prove continuity of $P$, we need to show that for each $\epsilon_{c}>0$, there exists $\delta_{c}>0$ such that $\left|x-x_{0}\right| \leq \delta_{c}$ implies $\left|P(x)-P\left(x_{0}\right)\right| \leq \epsilon_{c}$. Note that for each $x_{0} \in \mathcal{D}_{1}$, we have $P_{\dot{y}}\left(x_{0}\right)>0$ because of (5), hence continuity of $P$ can be proven without loss of generality by showing that for each $\epsilon_{c}<P_{\dot{y}}\left(x_{0}\right)$, a corresponding $\delta_{c}$ can be found by exploiting $(\tau, \epsilon)$-closeness. For $\phi^{x}=\left(\phi_{y}^{x}, \phi_{\dot{y}}^{x}, \phi_{u}^{x}\right),\left|P(x)-P\left(x_{0}\right)\right| \leq \epsilon_{c}$ is equivalent to $\left|\phi_{\dot{y}}^{x}\left(t_{3}, 2\right)-P_{\dot{y}}\left(x_{0}\right)\right| \leq \epsilon_{c}$, that is,

$$
\left|\phi_{\dot{y}}^{x}(s, 2)+\int_{s}^{t_{3}} \dot{\phi}_{\dot{y}}^{x}(\sigma, 2) d \sigma-P_{\dot{y}}\left(x_{0}\right)\right| \leq \epsilon_{c}
$$

by integrating from $(s, 2)$ in $(10)$ to $\left(t_{3}, 2\right)$, both in $\operatorname{dom} \phi^{x}$ (note that $(s, 2) \in \operatorname{dom} \phi^{x}$ implies $s \leq t_{3}$ by the definition of hybrid time domain). Since $(\tau, \epsilon)$-closeness holds for every $\epsilon>0$ and for the $\tau$ selected above, let us select suitably $\epsilon$ so that $\phi^{x}$, which is in an $\epsilon$-ball around $P\left(x_{0}\right)$ at some $(s, 2) \in \operatorname{dom} \phi^{x}$ as in $(10)$, reaches $\mathcal{D}_{1}$ before escaping the $\epsilon_{c}$-ball around $P\left(x_{0}\right)$ relative to continuity of $P$ in (11). For $\epsilon_{c}<P_{\dot{y}}\left(x_{0}\right)$, suitable bounds on the maximum magnitude of the acceleration in the $\epsilon_{c}$-ball and on the largest $t_{3}-s$ for $\phi^{x}$ to reach $\mathcal{D}_{1}$, provide indeed a positive $\epsilon<\epsilon_{c}$. Then, for the $\delta$ achieving $(\tau, \epsilon)$-closeness for $\tau$ above and such an $\epsilon, \delta_{c}=\delta$ guarantees $\left|P\left(x_{0}\right)-P(x)\right| \leq \epsilon_{c}$.

Energy injected at jumps and dissipated along flow.

For any point $x=(y, \dot{y}, u) \in \mathcal{C} \cup \mathcal{D}$, define its total energy as the sum of kinetic and potential energy, that is,

$$
e(x):=\frac{1}{2} m \dot{y}^{2}+U(y, u),
$$

which can be specialized for a point $x \in \mathcal{D}_{1}$ (that is, $x=\left(y_{1}, \dot{y}, u_{2}\right)$ for some $\left.\dot{y} \geq 0\right)$ as

$$
E(x):=\frac{1}{2} m \dot{y}^{2}+U\left(y_{1}, u_{2}\right) .
$$

In the sequel, for the convenience of the reader, we will use $e(x)$ for a generic $x \in \mathcal{C} \cup \mathcal{D}$ and $E(x)=e(x)$ whenever addressing points $x \in \mathcal{D}_{1}$. For each $x \in \mathcal{D}_{1}$, consider the maximal solution $\phi^{x}$. Because of Lemma $1, \phi^{x}$ is bound to jump from $\mathcal{D}_{1}$, flow for a finite time in $\mathcal{C}_{1}$, jump from $\mathcal{D}_{2}$ and flow for a finite time in $\mathcal{C}_{2}$ before reaching again $\mathcal{D}_{1}$. Such a solution and the jump times $t_{1}, t_{2}, t_{3}$ (defined in (6)), corresponding to the previous transitions, are depicted in Figure 4, to which the reader is referred for the rest of the proof. For such a portion of the solution up to $\left(t_{3}, 2\right)$, let us characterize the injected energy $I$ and the dissipated energy $D$ as a function of $x \in \mathcal{D}_{1}$.

Using the total energy definition in (12), the energy injected at the two jumps is for $x \in \mathcal{D}_{1}$

$$
\begin{aligned}
I:=\left[e\left(\phi^{x}\left(t_{1}, 1\right)\right)-e\left(\phi^{x}\left(t_{1}, 0\right)\right)\right] \\
\quad+\left[e\left(\phi^{x}\left(t_{2}, 2\right)\right)-e\left(\phi^{x}\left(t_{2}, 1\right)\right)\right] \\
=\left[U\left(y_{1}, u_{1}\right)-U\left(y_{1}, u_{2}\right)\right] \\
\quad+\left[U\left(y_{2}, u_{2}\right)-U\left(y_{2}, u_{1}\right)\right]=\text { constant }>0
\end{aligned}
$$

in accordance with the jump map (3b). The injected energy $I$ is a positive constant because $U\left(y_{1}, u_{1}\right)$ $U\left(y_{2}, u_{1}\right)>0$ and $U\left(y_{2}, u_{2}\right)-U\left(y_{1}, u_{2}\right)>0$ due to $(2 \mathrm{c})$.

As for the dissipated energy $D$, the derivative along solutions of the total energy $e$ in (12) is from (3a):

$$
\dot{e}=-c(y, \dot{y}) \dot{y}^{2} \text {. }
$$

Then, for each $x \in \mathcal{D}_{1}$ and the solution $\phi^{x}$, the dissipated energy $D: \mathcal{D}_{1} \rightarrow \mathbb{R}_{\geq 0}$ is

$$
\begin{aligned}
D(x):= & -\int_{t_{1}}^{t_{2}} \dot{e}\left(\phi^{x}(t, 1)\right) d t-\int_{t_{2}}^{t_{3}} \dot{e}\left(\phi^{x}(t, 2)\right) d t \\
= & \int_{t_{1}}^{t_{2}} c\left(\phi_{y}^{x}(t, 1), \phi_{\dot{y}}^{x}(t, 1)\right) \phi_{\dot{y}}^{x}(t, 1)^{2} d t \\
& \quad+\int_{t_{2}}^{t_{3}} c\left(\phi_{y}^{x}(t, 2), \phi_{\dot{y}}^{x}(t, 2)\right) \phi_{\dot{y}}^{x}(t, 2)^{2} d t
\end{aligned}
$$

where the integrals are restricted to the flow intervals.

Monotonicity of the dissipated energy D.

We can show that $D$ is strictly increasing with respect to the velocity of points in $\mathcal{D}_{1}$, that is,

$$
\left.\begin{array}{rl}
x_{a}=\left(y_{1}, \dot{y}_{a}, u_{2}\right) & \in \mathcal{D}_{1} \\
x_{b}=\left(y_{1}, \dot{y}_{b}, u_{2}\right) & \in \mathcal{D}_{1} \\
0 \leq \dot{y}_{a} & <\dot{y}_{b}
\end{array}\right\} \Longrightarrow D\left(x_{a}\right)<D\left(x_{b}\right),
$$

which we prove in two steps.

First, restate the integrals in (16) in terms of orbits. In each of the two integrals in (16), the time direction $j$ is fixed to $\bar{\jmath} \in\{1,2\}$. For the fixed $\bar{\jmath}$, we further split the computation of the corresponding integral in subintervals where $\phi_{\dot{y}}^{x}>0$ or $\phi_{\dot{y}}^{x}<0\left(\phi_{\dot{y}}^{x}(\cdot, \bar{\jmath})\right.$ can be zero only on a set of zero measure due to (5)). On each of such subintervals $\phi_{y}^{x}$ is an increasing or decreasing function of $t$, respectively, since $\dot{y}=\frac{d y}{d t}$. Then, on each one of such subintervals, $t \mapsto \phi_{y}^{x}(t, \bar{\jmath})$ is invertible with inverse $y \mapsto t(y)$, 


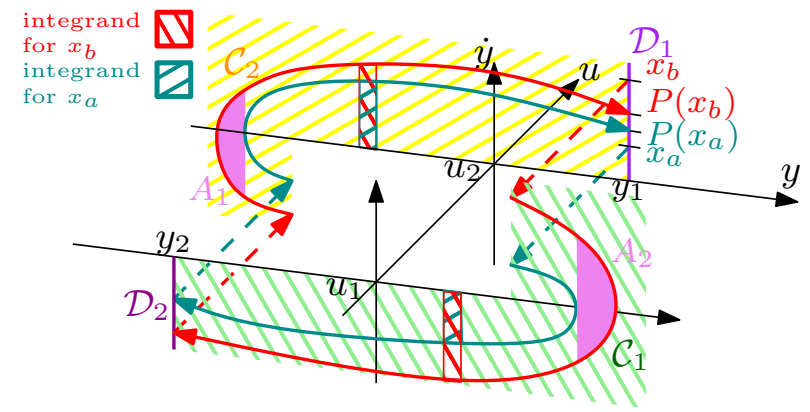

Figure 5. Integrand relative to the energy dissipated by damping for two orbits.

and $\frac{d t(y)}{d y}=\frac{1}{\left.\frac{d \phi_{y}^{x}(t, \bar{\jmath})}{d t}\right|_{t(y)}}=\frac{1}{\left.\phi_{\dot{y}}^{x}(t, \bar{\jmath})\right|_{t(y)}}$ (note that $\phi_{\dot{y}}^{x}(\cdot, \bar{\jmath})$ is absolutely continuous so that $\phi_{y}^{x}(\cdot, \bar{\jmath})$ is continuously differentiable). We can then change the integration variable from time $t$ to position $y$. For instance, for one such subinterval $\left[\tau_{a}, \tau_{b}\right]$ where either $\phi_{\dot{y}}^{x}>0$ or $\phi_{\dot{y}}^{x}<0$, the dissipated energy (taken positive as in (16)) is

$$
\begin{aligned}
& e\left(\phi^{x}\left(\tau_{a}, \bar{\jmath}\right)\right)-e\left(\phi^{x}\left(\tau_{b}, \bar{\jmath}\right)\right) \\
& =\int_{\tau_{a}}^{\tau_{b}} c\left(\phi_{y}^{x}(t, \bar{\jmath}), \phi_{\dot{y}}^{x}(t, \bar{\jmath})\right) \phi_{\dot{y}}^{x}(t, \bar{\jmath})^{2} d t \\
& =\int_{\phi_{y}^{x}\left(\tau_{a}, \bar{\jmath}\right)}^{\phi_{y}^{x}\left(\tau_{b}, \bar{\jmath}\right)} c\left(y, \phi_{\dot{y}}^{x}(t(y), \bar{\jmath})\right) \phi_{\dot{y}}^{x}(t(y), \bar{\jmath})^{2} \frac{d t(y)}{d y} d y \\
& =\int_{\phi_{y}^{x}\left(\tau_{a}, \bar{\jmath}\right)}^{\phi_{y}^{x}\left(\tau_{b}, \bar{\jmath}\right)} c\left(y, \phi_{\dot{y}}^{x}(t(y), \bar{\jmath})\right) \phi_{\dot{y}}^{x}(t(y), \bar{\jmath}) d y \\
& =\int_{\phi_{y}^{x}\left(\tau_{b}, \bar{\jmath}\right)}^{\phi_{y}^{x}\left(\tau_{a}, \bar{\jmath}\right)} c\left(y, \phi_{\dot{y}}^{x}(t(y), \bar{\jmath})\right)\left(-\phi_{\dot{y}}^{x}(t(y), \bar{\jmath})\right) d y,
\end{aligned}
$$

so that this integral is always positive regardless of the sign of $\phi_{\dot{y}}^{x}$ over $\left[\tau_{a}, \tau_{b}\right]\left(\phi_{\dot{y}}^{x}\right.$ negative implies $\phi_{y}^{x}\left(\tau_{b}, \bar{\jmath}\right)<$ $\left.\phi_{y}^{x}\left(\tau_{a}, \bar{\jmath}\right)\right)$. Consider also [10, Lemma 1] for a physical interpretation in terms of work when $c(y, \dot{y})$ is constant.

Second, consider $D\left(x_{a}\right)$ and $D\left(x_{b}\right)$ for $\dot{y}_{a}<\dot{y}_{b}$ as in (17). Take the two solution $\phi^{x_{a}}$ and $\phi^{x_{b}}$ with $\dot{y}_{a}<\dot{y}_{b}$ and split the integrals expressing the respective dissipated energies according to the integration principle just described. Up to reaching $\mathcal{D}_{1}$, the orbit of $\phi^{x_{a}}$ is in the interior of the area spanned by the orbit of $\phi^{x_{b}}$ (see Figure 5) because two orbits can not intersect during flow (if they did, uniqueness of solutions in Lemma 1 would be violated), and $y$ and $\dot{y}$ do not change across jumps. Due to this fact and Assumption (2b), when $\phi_{\dot{y}}$ is positive (cf. Figure 5 in $\mathcal{C}_{2}$ and Equation (18a)) the integrand $c\left(y, \phi_{\dot{y}}(t(y), \bar{\jmath})\right) \phi_{\dot{y}}(t(y), \bar{\jmath})$ relative to $\phi^{x_{a}}$ is strictly smaller than that of $\phi^{x_{b}}$. The same holds for $\phi_{\dot{y}}$ negative (cf. Figure 5 in $\mathcal{C}_{1}$ and Equation (18b)), besides the fact that the dissipated energy along $\phi^{x_{b}}$ has additional (positive) contributions (denoted by $A_{1}$ and $A_{2}$ in Figure 5). We conclude that the dissipated energy relative to $\phi^{x_{a}}$ is strictly less than that of $\phi^{x_{b}}$ as in (17).
Balance and uniqueness of equilibrium $P\left(x^{\star}\right)=x^{\star}$. Given the constant $I$ in (14), the monotonicity property in (17) (with respect to the velocity $\dot{y}$ of points $x \in \mathcal{D}_{1}$ ) implies that there exist a unique value of velocity $\dot{y}^{\star}$, and consequently a unique $x^{\star}=\left(y_{1}, \dot{y}^{\star}, u_{2}\right)$, such that

$$
D\left(x^{\star}\right)=I \text {. }
$$

We show now that $P\left(x^{\star}\right)=x^{\star}$, so that (9) is proven. Suppose by contradiction that $P\left(x^{\star}\right) \neq x^{\star}$, hence $E\left(x^{\star}\right) \neq E\left(P\left(x^{\star}\right)\right)$. Then, by the energy balance

$$
E\left(x^{\star}\right)+I-D\left(x^{\star}\right)=E\left(P\left(x^{\star}\right)\right),
$$

we can deduce $D\left(x^{\star}\right) \neq I$, which is a contradiction.

Asymptotic stability.

For the global asymptotic stability of the unique equilibrium $x^{\star}$ of $(8)$, consider the Lyapunov function $V: \mathcal{D}_{1} \rightarrow$ $\mathbb{R}_{\geq 0}$ defined for $x=\left(y_{1}, \dot{y}, u_{2}\right)$ and $x^{\star}=\left(y_{1}, \dot{y}^{\star}, u_{2}\right)$ as

$$
V(x):=\left|x-x^{\star}\right|^{2}=\left(\dot{y}-\dot{y}^{\star}\right)^{2} .
$$

Because in (8) $P$ is continuous and $\mathcal{D}_{1}$ is closed (so it satisfies the hybrid basic conditions [18, Assumption 6.5]) and the attractor is the point $x^{\star}$, we need to prove:

$$
V(P(x))-V(x)<0 \quad \forall x \in \mathcal{D}_{1}, x \neq x^{\star},
$$

or, equivalently,

$$
\left(\dot{y}^{+}-\dot{y}\right)\left(\dot{y}^{+}-\dot{y}^{\star}+\dot{y}-\dot{y}^{\star}\right)<0 \quad \forall \dot{y} \geq 0, \dot{y} \neq \dot{y}^{\star}
$$

which is obtained by substituting (21) and using $\left(y_{1}, \dot{y}^{+}, u_{2}\right)=P(x)$ from (8). Property (22) is true if the following implications hold

$$
\begin{aligned}
& \dot{y}^{+}>\dot{y} \Longrightarrow \dot{y}^{\star}>\dot{y}^{+}>\dot{y} \\
& \dot{y}^{+}<\dot{y} \Longrightarrow \dot{y}^{\star}<\dot{y}^{+}<\dot{y}
\end{aligned}
$$

Indeed, since $\dot{y}^{+}=\dot{y}$ holds true only for $\dot{y}=\dot{y}^{\star}$ due to (9), either (23a) or (23b) holds, under either of which (22) is true. To conclude the proof, we then show the validity of (23). To prove (23a), we just need to prove $\dot{y}^{+}>\dot{y} \Longrightarrow \dot{y}^{\star}>\dot{y}^{+} \cdot \dot{y}^{+}>\dot{y}$ implies $E(P(x))>E(x)$ by (13). From an energy balance $E(P(x))-E(x)=I-D(x)$, so $I-D(x)>0$. By $(17)$ and (19), $I-D(x)>0$ implies $\dot{y}^{\star}>\dot{y}$. Consider the two solutions $\phi^{x^{\star}}$ and $\phi^{x}$. Due to (3b), the velocity remains the same across jumps, so $\dot{y}^{\star}=\phi_{\dot{y}}^{x^{\star}}(0,1)>\phi_{\dot{y}}^{x}(0,1)=\dot{y}$. While flowing in $\mathcal{C}_{1}$, uniqueness of solutions from Lemma 1 holds, meaning that the orbits of the two solutions cannot intersect. So, after the flow in $\mathcal{C}_{1}$, $\phi_{\dot{y}}^{x^{\star}}\left(t_{2}^{x^{\star}}, 1\right)<\phi_{\dot{y}}^{x}\left(t_{2}^{x}, 1\right)$ where the dependence on $x$ or $x^{\star}$ of $t_{2}$ (cf. (6)) was made explicit for clarity. Repeating the reasoning for $\mathcal{D}_{2}$ and $\mathcal{C}_{2}$, we obtain finally that $\dot{y}^{\star}=\phi_{\dot{y}}^{x^{\star}}\left(t_{3}^{x^{\star}}, 2\right)>\phi_{\dot{y}}^{x}\left(t_{3}^{x}, 2\right)=\dot{y}^{+} .(23 \mathrm{~b})$ is proven with 
parallel considerations to those for (23a).

We proved in Lemma 1 that all solutions jump from $\mathcal{D}_{1}$ after some finite hybrid time and we proved in Lemma 2 that there exists a unique $x^{\star} \in \mathcal{D}_{1}$ such that $P\left(x^{\star}\right)=x^{\star}$. The unique periodic orbit starting from $x^{\star}:=\left(y_{1}, \dot{y}^{\star}, u_{2}\right)$ has a finite continuous period $T>0$ and a discrete period $J=2$, as from Definition 2 , and defines a compact attractor $\mathcal{A}$ as

$$
\mathcal{A}:=\left\{x: x=\phi^{x^{\star}}(t, j) \text { for some }(t, j) \in \operatorname{dom} \phi^{x^{\star}}\right\} .
$$

Therefore, the existence of a unique nontrivial periodic orbit that is globally asymptotically stable (Theorem 1) is an immediate consequence of the next proposition.

Proposition $1 \mathcal{A}$ in (24) is (robustly) globally asymptotically stable.

Proof of Proposition 1. Stability follows from Lemma 2 (stability of $x^{\star}$ ) and continuity of solutions on compact time intervals, as established in [18, Proposition 6.14]. Indeed, all solutions starting in a neighborhood of $x^{\star}$ come back to $\mathcal{D}_{1}$ after a uniform finite time, and their distance from the solution starting at $x^{\star}$ (namely, an upper bound of their distance to $\mathcal{A}$ because that solution only evolves in $\mathcal{A}$ ) is a continuous function of the initial distance from $x^{\star}$. As a consequence for each $\epsilon>0$ one can pick a small enough $\delta$ neighborhood of the solution starting from $x^{\star}$ from which the ensuing solutions remain $\epsilon$ close to $\mathcal{A}$. Global attractivity follows from Lemma 2 (attractivity of $x^{\star}$ ), persistent jumping in Lemma 1 and again continuity of solutions on compact time intervals. These properties are also uniform, as established in [18, Theorem 7.12], and robust, as established in [18, Theorem 7.21], because $\mathcal{A}$ is compact and the hybrid basic conditions [18, Assumption 6.5] are satisfied.

\section{Reset-damped oscillations}

\subsection{General theory}

Continuing the discussion of Section 2.1, we show that for the nonlinear mechanical system (1a), that is,

$$
m \ddot{y}+c(y, \dot{y}) \dot{y}+\frac{\partial U}{\partial y}(y, u)=0
$$

simple reset laws can also be used to damp oscillations, by increasing the natural dissipation of the system. In particular, we assume that (25a) has a globally asymptotically stable equilibrium point $\left(y_{0}, 0\right)$ when the input is at rest $\left(u=u_{0}\right)$, so that a faster damping of the oscillations through resets accelerates the convergence to this attractor.

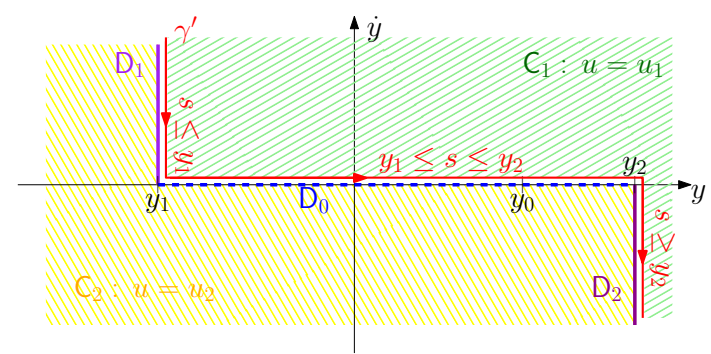

Figure 6. Reset law in (25): relevant quantities and sets.

The damping of the oscillations is obtained by a reset law similar to the one adopted in Section 2. Based on Figure 6, a suitable mirroring $\gamma^{\prime}$ of the reset curve $\gamma$ in (1b) induces earlier reset instants, with the goal of extracting energy from the system. Then, $u$ is determined according to

$$
\begin{aligned}
u & = \begin{cases}u_{1} & \text { if }(y, \dot{y}) \in \mathrm{C}_{1} \\
u_{2} & \text { if }(y, \dot{y}) \in \mathrm{C}_{2}\end{cases} \\
\mathrm{C}_{1} & :=\left\{(y, \dot{y}):\left(\dot{y}>0, y_{1}<y \leq y_{2}\right) \text { or } y>y_{2}\right\} \\
\mathrm{C}_{2} & :=\left\{(y, \dot{y}):\left(\dot{y}<0, y_{1} \leq y<y_{2}\right) \text { or } y<y_{1}\right\} .
\end{aligned}
$$

Resets are triggered when the state $(y, \dot{y})$ crosses the branches

$$
\begin{aligned}
& \mathrm{D}_{1}:=\left\{(y, \dot{y}): y=y_{1}, \dot{y} \geq 0\right\} \\
& \mathrm{D}_{2}:=\left\{(y, \dot{y}): y=y_{2}, \dot{y} \leq 0\right\} \\
& \mathrm{D}_{0}:=\left\{(y, \dot{y}): y_{1} \leq y \leq y_{2}, \dot{y}=0\right\} .
\end{aligned}
$$

When $D_{1}$ or $D_{2}$ are crossed, the control $u$ toggles between $u_{1}$ and $u_{2}$, like in Section 2. The reset law in (25b) is similar to the reset band considered in $[4,5]$. On the other hand, when the branch $D_{0}$ is crossed, the system resets its actuation $u$ to the rest value $u_{0}$, which globally stabilizes the equilibrium $\left(y_{0}, 0\right)$, with $y_{1}<y_{0}<y_{2}$, from any point of $C_{0}:=\mathbb{R}^{2}$. To ensure global asymptotic stability of $\left(y_{0}, 0\right)$ when $u=u_{0}$ is applied, we need the following sector assumption (26a) on the potential. Moreover, (26b)-(26c) guarantee a decrease in the potential energy when crossing the branch $D_{0}$, that is, resetting from $u_{1}$ to $u_{0}$ or from $u_{2}$ to $u_{0}$.

\section{Assumption 2 Given $y_{1}<y_{0}<y_{2}$,}

$$
\left.\begin{array}{c}
\left(y-y_{0}\right) \frac{\partial U}{\partial y}\left(y, u_{0}\right)>0 \text { for } y \neq y_{0} \\
U\left(y, u_{0}\right)-U\left(y, u_{1}\right)<0 \\
U\left(y, u_{0}\right)-U\left(y, u_{2}\right)<0
\end{array}\right\} \text { for all } y \in\left[y_{1}, y_{2}\right]
$$

Proposition 2 establishes that each reset sequence causes a nonzero decrease of the total energy of the system, as compared to the case with no actuation $\left(u=u_{0}\right)$. Draining energy from the system helps achieving faster damping of oscillations. 


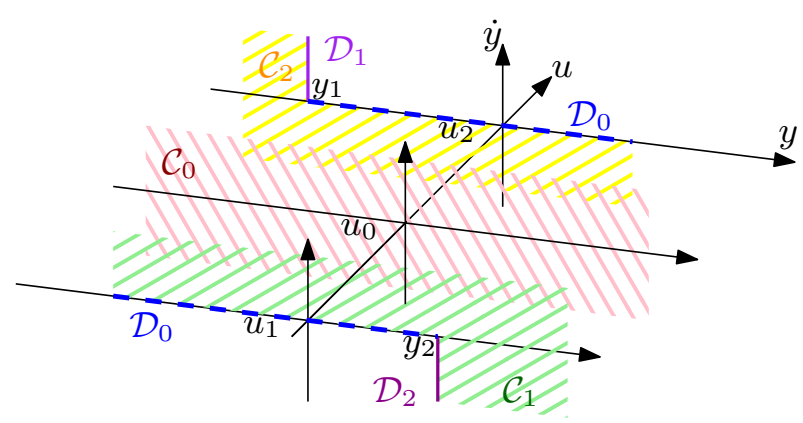

Figure 7. Hybrid formulation (27) of system (25).

Proposition 2 Under Assumptions 1 and 2, each solution to (25) undergoes a finite number of resets (jumps) that induce a nonzero decrease of the total energy defined in (12). In particular, for each $\bar{e}>0$, there exists $N(\bar{e})$ such that all solutions with initial total energy smaller than $\bar{e}$ perform at most $N(\bar{e})$ jumps.

Remark 3 Assumptions (26b)-(26c) are required to guarantee a nonzero decrease for each reset sequence (in particular for the tail of the sequence). Higher energy levels $\bar{e}$ induce more jumps $N(\bar{e})$. An upper bound on $N(\bar{e})$ is explicitly derived at the end of the proof of Proposition 2, as a function of the bound $\delta$ on the dissipated energy at jumps in (31).

Proposition 2 clarifies the desirable features of the proposed law, but also constitutes a key ingredient for proving Theorem 2, which establishes that the introduction of the resets preserves asymptotic stability.

Theorem 2 For the mechanical system (25) satisfying Assumptions 1 and 2, $\left(y_{0}, 0\right)$ is a globally asymptotically stable equilibrium point.

\subsection{Hybrid formulation and proofs}

As we did in Section 2.2 for system (1), let us reformulate system (25) as a hybrid dynamical system:

$$
\begin{aligned}
& \left.\begin{array}{l}
m \ddot{y}+c(y, \dot{y}) \dot{y}+\frac{\partial U}{\partial y}(y, u)=0 \\
\dot{u}=0
\end{array}\right\} \quad(y, \dot{y}, u) \in \mathcal{C} \\
& \left.\begin{array}{l}
y^{+}=y \\
\dot{y}^{+}=\dot{y} \\
u^{+}=u_{i}
\end{array}\right\} \quad(y, \dot{y}, u) \in \mathcal{D}_{i}, i \in\{0,1,2\} \\
& \mathcal{C}:=\left(\overline{\mathrm{C}}_{1} \times\left\{u_{1}\right\}\right) \cup\left(\overline{\mathrm{C}}_{2} \times\left\{u_{2}\right\}\right) \cup\left(\overline{\mathrm{C}}_{0} \times\left\{u_{0}\right\}\right) \\
& =: \mathcal{C}_{1} \cup \mathcal{C}_{2} \cup \mathcal{C}_{0} \\
& \mathcal{D}:=\bigcup_{i \in\{1,2,3\}} \mathcal{D}_{i} \quad \text { with } \begin{cases}\mathcal{D}_{1} & :=\mathrm{D}_{1} \times\left\{u_{2}\right\} \\
\mathcal{D}_{2} & :=\mathrm{D}_{2} \times\left\{u_{1}\right\} \\
\mathcal{D}_{0} & :=\mathrm{D}_{0} \times\left\{u_{1}, u_{2}\right\} .\end{cases}
\end{aligned}
$$

We first prove Proposition 2 aided by Figure 7 .
Proof of Proposition 2. We divide the proof in steps. Variation of total energy across jumps.

Using the definition of total energy in (12), we define its variations across jumps for all $y \in\left[y_{1}, y_{2}\right]$

$$
\begin{aligned}
\Delta_{1 \rightarrow 2} & :=e\left(\left(y_{2}, \dot{y}, u_{2}\right)\right)-e\left(\left(y_{2}, \dot{y}, u_{1}\right)\right) \\
& =U\left(y_{2}, u_{2}\right)-U\left(y_{2}, u_{1}\right) \\
\Delta_{2 \rightarrow 1} & :=e\left(\left(y_{1}, \dot{y}, u_{1}\right)\right)-e\left(\left(y_{1}, \dot{y}, u_{2}\right)\right) \\
& =U\left(y_{1}, u_{1}\right)-U\left(y_{1}, u_{2}\right) \\
\Delta_{1 \rightarrow 0}(y) & :=e\left(\left(y, 0, u_{0}\right)\right)-e\left(\left(y, 0, u_{1}\right)\right) \\
& =U\left(y, u_{0}\right)-U\left(y, u_{1}\right) \\
\Delta_{2 \rightarrow 0}(y) & :=e\left(\left(y, 0, u_{0}\right)\right)-e\left(\left(y, 0, u_{2}\right)\right) \\
& =U\left(y, u_{0}\right)-U\left(y, u_{2}\right),
\end{aligned}
$$

where each of these $\Delta_{i \rightarrow j}$ is the variation due to a jump that resets the control from $u_{i}$ to $u_{j}$. Assumption 1 (Equation (2c)) implies that for all $y \in\left(y_{1}, y_{2}\right)$

$$
\begin{aligned}
& U\left(y_{1}, u_{1}\right)<U\left(y, u_{1}\right)<U\left(y_{2}, u_{1}\right) \\
& U\left(y_{2}, u_{2}\right)<U\left(y, u_{2}\right)<U\left(y_{1}, u_{2}\right) .
\end{aligned}
$$

Using the relations in (28), we can obtain that for all $y \in\left[y_{1}, y_{2}\right]$ (the equation number over the inequality sign justifies the corresponding bound)

$$
\begin{aligned}
& \Delta_{1 \rightarrow 2}+\Delta_{2 \rightarrow 1} \stackrel{(29 a),(29 b)}{<} 0 \\
& \Delta_{1 \rightarrow 2}+\Delta_{2 \rightarrow 0}(y) \stackrel{(29 \mathrm{~b})}{\leq} U\left(y, u_{0}\right)-U\left(y_{2}, u_{1}\right) \\
& \stackrel{(29 \mathrm{a})}{\leq} U\left(y, u_{0}\right)-U\left(y, u_{1}\right)=\Delta_{1 \rightarrow 0}(y) \stackrel{(26 \mathrm{~b})}{<} 0 \\
& \Delta_{2 \rightarrow 1}+\Delta_{1 \rightarrow 0}(y) \stackrel{(29 a)}{\leq} U\left(y, u_{0}\right)-U\left(y_{1}, u_{2}\right) \\
& \stackrel{(29 \mathrm{~b})}{\leq} U\left(y, u_{0}\right)-U\left(y, u_{2}\right)=\Delta_{2 \rightarrow 0}(y) \stackrel{(26 \mathrm{c})}{<} 0
\end{aligned}
$$

In light of (30), a scalar $\delta>0$ can then be found such that for all $y \in\left[y_{1}, y_{2}\right]$

$$
\begin{aligned}
& \Delta_{1 \rightarrow 2}+\Delta_{2 \rightarrow 1}<-\delta \\
& \Delta_{1 \rightarrow 2}+\Delta_{2 \rightarrow 0}(y) \leq \Delta_{1 \rightarrow 0}(y)<-\delta \\
& \Delta_{2 \rightarrow 1}+\Delta_{1 \rightarrow 0}(y) \leq \Delta_{2 \rightarrow 0}(y)<-\delta
\end{aligned}
$$

Finite number $N(\bar{e})$ of jumps and nonzero decrease of total energy.

If solutions to $(27)$ start in $\mathcal{C}_{0}$, no jumps are allowed because of $(27 \mathrm{~d})$ and $u=u_{0}$. In this case, the jumps are clearly finite.

Solutions in $\mathcal{D} \cap \mathcal{C}$ can only jump because any flowing solution along the vector field (27a) would flow outside $\mathcal{C}$. Indeed,

- $\dot{y}<0$ in $\left(\mathcal{D}_{2} \cap \mathcal{C}_{1}\right) \backslash\left\{\left(y_{2}, 0, u_{1}\right)\right\}$, and then solutions would flow to the forbidden set $y<y_{2}$;

- $\ddot{y}=-\frac{1}{m} \frac{\partial U}{\partial y}\left(y, u_{1}\right)<0$ in $\mathcal{D}_{0} \cap \mathcal{C}_{1}$ due to (5) and then 
forbidden flow to $\dot{y}<0, y<y_{2}$ would occur.

Analogous arguments hold in $\mathcal{D} \cap \mathcal{C}_{2}$.

Consider now a maximal solution starting in $\mathcal{C}_{1}$ (a parallel argument holds when starting in $\mathcal{C}_{2}$ ). The solution is bound to reach (and jump from) either $\mathcal{D}_{0}$ or $\mathcal{D}_{2}$ after a finite time because $(i) \ddot{y}<0$ in $\mathcal{C}_{1} \cap\left\{\left(y, \dot{y}, u_{1}\right): \dot{y} \geq 0\right\}$ due to (5) and (ii) $\dot{y}<0$ elsewhere in $\mathcal{C}_{1}$ (see also step (b) in the proof of Lemma 1). Given this fact, assume by contradiction that the number of jumps is not finite. This is only possible if there exists a complete solution $\phi_{c}$ that keeps jumping from $\mathcal{D}_{1}$ and $\mathcal{D}_{2}$, but never from $\mathcal{D}_{0}$ because from $\mathcal{D}_{0}$ solutions can only jump to $\mathcal{C}_{0}$. Take any jump time $t_{j}$ as defined in (6) such that $\phi_{c}\left(t_{j}, j-1\right) \in$ $\mathcal{D}_{1}$. Then we have $\phi_{c}\left(t_{j}, j\right) \in \mathcal{C}_{1}, \phi_{c}\left(t_{j+1}, j\right) \in \mathcal{D}_{2}$, $\phi_{c}\left(t_{j+1}, j+1\right) \in \mathcal{C}_{2}$ and $\phi_{c}\left(t_{j+2}, j+1\right) \in \mathcal{D}_{1}$ (refer to Figure 7), and the following holds:

$$
\begin{aligned}
& e\left(\phi_{c}\left(t_{j+2}, j+1\right)\right)-e\left(\phi_{c}\left(t_{j}, j-1\right)\right) \\
& =e\left(\phi_{c}\left(t_{j}, j\right)\right)-e\left(\phi_{c}\left(t_{j}, j-1\right)\right) \\
& \quad+\int_{t_{j}}^{t_{j+1}} \frac{d}{d t} e\left(\phi_{c}(t, j)\right) d t \\
& +e\left(\phi_{c}\left(t_{j+1}, j+1\right)\right)-e\left(\phi_{c}\left(t_{j+1}, j\right)\right) \\
& \quad+\int_{t_{j+1}}^{t_{j+2}} \frac{d}{d t} e\left(\phi_{c}(t, j+1)\right) d t \\
& \leq \Delta_{2 \rightarrow 1}+\Delta_{1 \rightarrow 2}<-\delta,
\end{aligned}
$$

where we used (15) and (2a) for bounding the integrals, and then (31a). Equation (32) shows that such a "bad" solution $\phi_{c}$ cannot exist because the persistent jumping from $\mathcal{D}_{1}$ would decrease arbitrarily the total energy $e$ associated with $\phi_{c}$ (when it crosses $\mathcal{D}_{1}$ ), and this contradicts $e(x)=\frac{1}{2} m \dot{y}^{2}+U\left(y_{1}, u_{2}\right) \geq U\left(y_{1}, u_{2}\right)$ which holds in $\mathcal{D}_{1}$.

Equation (32) also holds for a solution jumping a finite number of times from $\mathcal{D}_{1}$ and the same number of times from $\mathcal{D}_{2}$. After that, solutions can jump a next to last time from $\mathcal{D}_{1}$ or from $\mathcal{D}_{2}$, and then they jump from $\mathcal{D}_{0}$. Note that the energy also decreases by at least $-\delta$ in these tail jumps as established by (31b) and (31c). This implies that for each $\bar{e}$, all solutions with initial total energy smaller than $\bar{e}$ perform at most $N(\bar{e}) \leq 2 \frac{\bar{e}}{\delta}+3$ jumps.

To prove Theorem 2, we use the following fact, which is a consequence of [17, Theorem 31], when all jumps are treated as events.

Fact 1 ([17, Theorem 31]) Suppose that the hybrid system $\mathcal{H}=(\mathcal{C}, F, \mathcal{D}, G)$ with state $x \in \mathbb{R}^{n}$ satisfies the Basic Assumptions (see [17, p. 43]). Let the compact set $\mathcal{A} \subset \mathbb{R}^{n}$ satisfy $G(\mathcal{D} \cap \mathcal{A}) \subset \mathcal{A}$, and assume that $\mathcal{A}$ is globally asymptotically stable for the hybrid system with no jumps $\mathcal{H}^{0}=(\mathcal{C}, F, \emptyset, \emptyset)$. Also suppose that, for the hybrid system $\mathcal{H}$ and each compact set $\mathcal{K} \subset \mathbb{R}^{n}$, there exists $N>0$ such that each solution starting in $\mathcal{K}$ experiences no more than $N$ jumps. Then the set $\mathcal{A}$ is globally asymptotically stable for the system $\mathcal{H}$.

Proof. To prove the fact, we simply need to find a suitable outer semicontinuous event indicator $\mathcal{E}$ such that $\mathcal{D}^{0}$ and $G^{0}$ in [17, Theorem 31] both coincide with the empty set. Setting $\mathcal{E}(g, x):=\emptyset$ for all $x \in \mathcal{D}, g \in G(x)$, results in an event indicator making $G^{0}=\emptyset$ and $\mathcal{D}^{0}=\emptyset$ as from [17, Equations (38)-(39)]. Note that since in [17] an event is a pair $(g, x) \in \mathbb{R}^{n} \times \mathbb{R}^{n}$ such that $\mathcal{E}(g, x)=\emptyset$, our selection of $\mathcal{E}$ identifies any jump with an event.

Proof of Theorem 2. The assumptions of Fact 1 are verified by $(27)$ and $\mathcal{A}:=\left\{\left(y_{0}, 0, u_{0}\right)\right\}$, so the proof of this theorem follows from concatenating Fact 1 with Proposition 2 , after noting that in each compact set $\mathcal{K}$ there is a maximum value $\bar{e}$ of the total energy.

\section{Applications}

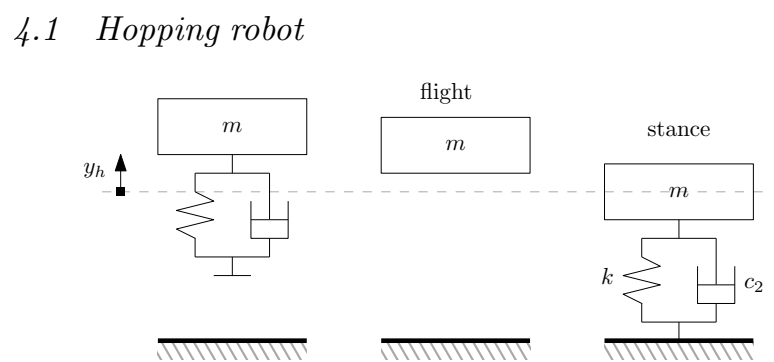

Figure 8. Hopping robot on a fixed spot.

Consider the hopping robot in Figure 8, described by position $y_{h}$ and velocity $\dot{y}_{h}$ and acted upon by the piecewise constant input $u$. The standard hopping behavior of the robot is defined by two main phases. During the stance phase the robot is attached to the ground and follows the dynamics of an oscillator. During the flight phase the robot is no longer attached to the ground and follows a ballistic motion. The transitions from stance to flight and from flight to stance correspond approximately to the spring being undeformed after having been compressed during the stance, and to the bottom end of the spring touching the ground after the flight, respectively. As in [28], the hopper is equipped with a motor that preloads the spring during the flight phase by a length $\theta$ (we assume that the duration of the flight phase allows for the spring to be shortened and preloaded). This storage of energy is then released via a clutch mechanism at the contact with the ground.

The preceding physical description motivates us to use the formulation in Section 2.1 and write a simplified model of the hopper. We specialize the reset curve $\gamma$ 
in (1b) by setting $y_{1}=\theta-\epsilon$ and $y_{2}=0$. The curve divides the space $\left(y_{h}, \dot{y}_{h}\right)$ into the regions

$$
\begin{aligned}
& \mathrm{C}_{1}:=\left\{\left(y_{h}, \dot{y}_{h}\right): y_{h}>\theta-\epsilon\right. \\
& \text { or } \left.\left(\dot{y}_{h}<0,0<y_{h} \leq \theta-\epsilon\right)\right\} \\
& \mathrm{C}_{2}:=\left\{\left(y_{h}, \dot{y}_{h}\right): y_{h}<0\right. \\
& \text { or } \left.\left(\dot{y}_{h}>0,0 \leq y_{h}<\theta-\epsilon\right)\right\} \text {, }
\end{aligned}
$$

where $C_{1}$ correspond to the flight, and $C_{2}$ to the stance. The (small) parameter $\epsilon>0$ is introduced to take into account uncertainties in the transition from stance to flight. The case $\epsilon=0$ corresponds to this transition occuring exactly when the spring is undeformed after being loaded during stance. In practice the system enters into ballistic motion even if the spring has not fully extended yet. Our model does not capture this level of detail but the parameter $\epsilon>0$ is used to model the anticipation of the transition from stance to flight. In the corresponding flow regions the input takes the values

$$
u= \begin{cases}0 & \text { if }\left(y_{h}, \dot{y}_{h}\right) \in \mathrm{C}_{1} \\ \theta & \text { if }\left(y_{h}, \dot{y}_{h}\right) \in \mathrm{C}_{2}\end{cases}
$$

Resets are triggered when crossing the branches

$$
\begin{aligned}
& \mathrm{D}_{1}:=\left\{\left(y_{h}, \dot{y}_{h}\right): y_{h}=\theta-\epsilon, \dot{y}_{h} \geq 0\right\} \\
& \mathrm{D}_{2}:=\left\{\left(y_{h}, \dot{y}_{h}\right): y_{h}=0, \dot{y}_{h} \leq 0\right\}
\end{aligned}
$$

Finally, the continuous evolution in (1a) during flight $\left(u=u_{1}=0\right.$ and potential $\left.y_{h} \mapsto U\left(y_{h}, u_{1}\right)=m g y_{h}\right)$ becomes

$$
m \ddot{y}_{h}+c_{1} \dot{y}_{h}+m \mathrm{~g}=0,
$$

where $m$ is the mass, $\mathrm{g}$ is the gravity, and $c_{1}$ is the (small) air friction. During stance $\left(u=u_{2}=\theta\right.$ and potential $\left.y_{h} \mapsto U\left(y_{h}, u_{2}\right)=\frac{1}{2} k\left(y_{h}-\theta\right)^{2}+m g y_{h}\right)$, (1a) becomes

$$
m \ddot{y}_{h}+c_{2} \dot{y}_{h}+k\left(y_{h}-\theta\right)+m \mathrm{~g}=0
$$

where $k$ is the stiffness, and $c_{2}$ combines possibly an actual mechanical damper, the structural damping and the dissipation occuring at the impact, so that typically $c_{2} \gg c_{1}$. Note that (33f) and (33g) can be merged into the single equation $m \ddot{y}_{h}+c\left(y_{h}, \dot{y}_{h}\right) \dot{y}_{h}+\frac{\partial U}{\partial y_{h}}\left(y_{h}, u\right)=0$ where the nonlinear damping $c(\cdot, \cdot)$ can be defined in $\bar{C}_{1}$ and $\overline{\mathrm{C}}_{2}$ based on $c_{1}$ and $c_{2}$, and $\frac{\partial U}{\partial y_{h}}$ based on the two above potentials $y_{h} \mapsto U\left(y_{h}, u_{1}\right)$ and $y_{h} \mapsto U\left(y_{h}, u_{2}\right)$. The control $u$ is determined by (33c).

For $\epsilon>0$, consider a spring stiffness $k$ such that $k \epsilon>m \mathrm{~g}$. Then, Assumption 1 is satisfied since for $(2 \mathrm{c})$, $\frac{\partial U}{\partial y_{h}}\left(y_{h}, u_{2}\right)=k\left(y_{h}-\theta\right)+m \mathrm{~g}<0$ in $\overline{\mathrm{C}}_{2}$ (note also that $c(\cdot, \cdot)$ above is differentiable in each $\overline{\mathrm{C}}_{1}$ and $\overline{\mathrm{C}}_{2}$ and satisfies $(2 \mathrm{a})-(2 \mathrm{~b})$, and that $\frac{\partial U}{\partial y_{h}}$ is locally Lipschitz in each $\overline{\mathrm{C}}_{1}$ and $\overline{\mathrm{C}}_{2}$, as required in Assumption 1). Therefore Theorem 1 guarantees that the hopper has a unique nontrivial periodic orbit which is asymptotically stable. We illustrate this result in the following example.

Example 2 Consider for (33) the parameters $m=$ $50 \mathrm{~kg}, c_{1}=5 \mathrm{Ns} / \mathrm{m}, c_{2}=400 \mathrm{Ns} / \mathrm{m}, k=100 \mathrm{kN} / \mathrm{m}$, $\mathrm{g}=9.81 \mathrm{~m} / \mathrm{s}^{2}, \theta=0.1 \mathrm{~m}, \epsilon=0.005 \mathrm{~m}$. The stance phase begins when the solution crosses the dashed threshold at 0 downwards, and ends when it crosses the dashed threshold at $\theta-\epsilon$ upwards. The periodic orbit (in light blue) arises when the energy injected by the spring preload (and released through a clutch mechanism when touching ground as in [28]) balances the energy dissipated along the orbit.
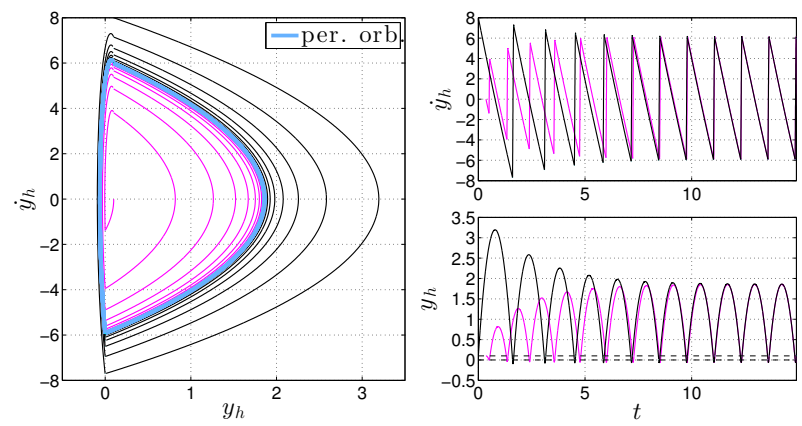

Figure 9. Hopping robot. Left: phase portrait with the periodic solution (light blue) and two other solutions (magenta, black) starting inside and outside the periodic orbit. Right, bottom: time evolution of $y_{h}$ with the position thresholds 0 and $\theta-\epsilon$ (dashed) determining the transitions between flight and stance. Right, top: time evolution of $\dot{y}_{h}$.

\subsection{Automotive suspension}

Consider the simplified one-degree-of-freedom model of an automotive suspension represented on the left of Figure 10 with a sprung mass $m_{s}$, where $y_{s}$ is the displacement of the sprung mass from its equilibrium position, $\dot{y}_{s}$ is the corresponding velocity, $y_{r}$ is the displacement of the road and the parameters $k_{s}$ and $c_{s}$ are the stiffness and damping relative to the suspension. The corresponding model is

$$
m_{s} \ddot{y}_{s}+c_{s}\left(\dot{y}_{s}-\dot{y}_{r}\right)+k_{s}\left(y_{s}-y_{r}-u\right)=0,
$$

where we first consider a zero $y_{r}$ to illustrate Section 3.1 and then, in Example 3, a nonzero $y_{r}$ for a more realistic two-degree-of-freedom suspension. The quantity $u$
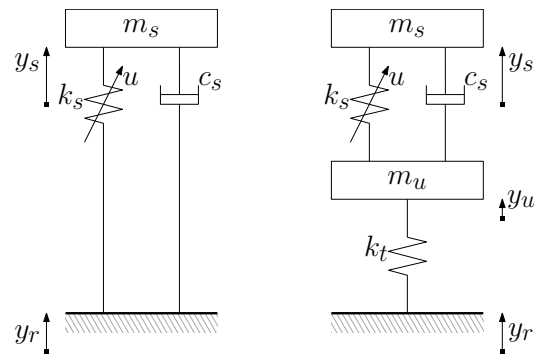

Figure 10. Simplified and full quarter car model. 

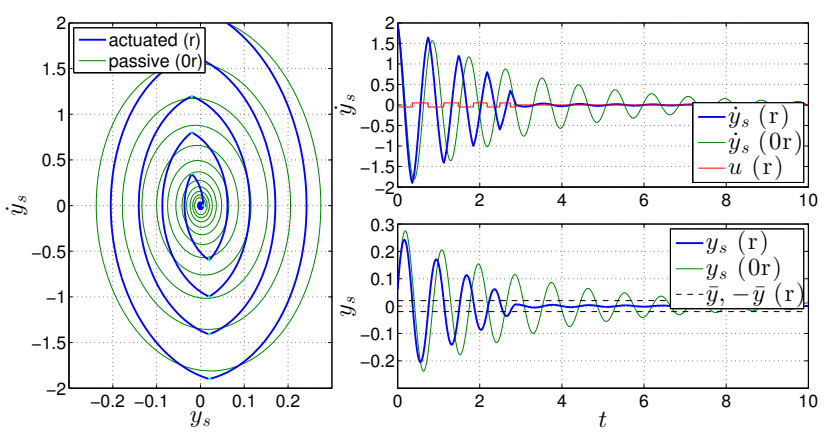

Figure 11. Left: phase portrait for the actuated and passive $(u(t)=0$ for all $t)$ suspension. Right, bottom: time evolution of $y_{s}$. Right, top: time evolution of $\dot{y}_{s}$ and $u$ for the actuated suspension.

denotes the reset action in terms of spring preload that is chosen according to Section 3.1, with the symmetric selections

$$
\begin{aligned}
& u_{2}=-u_{1}=\bar{u}>0 \\
& y_{2}=-y_{1}=\bar{y}>0 .
\end{aligned}
$$

The rest value of $u$ is $u_{0}=0$, which is applied after crossing the set $\mathrm{D}_{0}=\left\{\left(y_{s}, \dot{y}_{s}\right):-\bar{y} \leq y_{s} \leq \bar{y}, \dot{y}_{s}=0\right\}$ and is associated to the equilibrium $y_{0}=0$ of the free dynamics.

Under the condition $\bar{y}<\frac{\bar{u}}{2}$, Assumptions 1 and 2 are satisfied, and Theorem 2 establishes that the equilibrium $(0,0)$ of the reset suspension is globally asymptotically stable.

The simple reset actuation guarantees that energy is drained from the system at each reset, as per Proposition 2: note that $\bar{u}>0, \bar{y}>0$ suffice to drain energy when resetting from $D_{1}$ to $D_{2}$, or vice versa, since in both these resets the drained energy is $-\frac{1}{2} k(2 \bar{u})(2 \bar{y})$. This improves the suspension performance compared to the case when no actuation is present $(u(t)=0$ for all nonnegative $t$ ), as we illustrate in the following example.

Example 3 Fix the parameters $m_{s}=400 \mathrm{~kg}, c_{s}=$ $260 \mathrm{Ns} / \mathrm{m}, k_{s}=20 \cdot 10^{3} \mathrm{~N} / \mathrm{m}, \bar{u}=0.05 \mathrm{~m}, \bar{y}=0.02 \mathrm{~m}$ in (34). Figure 11 shows the improvement on the unactuated suspension (green) obtained by the reset one (blue). Note that for the actuated suspension the segment $D_{0}$ is intersected at about $2.9 \mathrm{~s}$ and afterwards the mechanical system evolves according to its free dynamics.

We also show that the proposed reset law can be suitably applied to the complete quarter car model [41, Chapter 3.1] on the right of Figure 10, where $y_{u}$ is the displacement of the unsprung mass $m_{u}$ from its equilibrium position, $\dot{y}_{u}$ is the corresponding velocity and $k_{t}$ is the
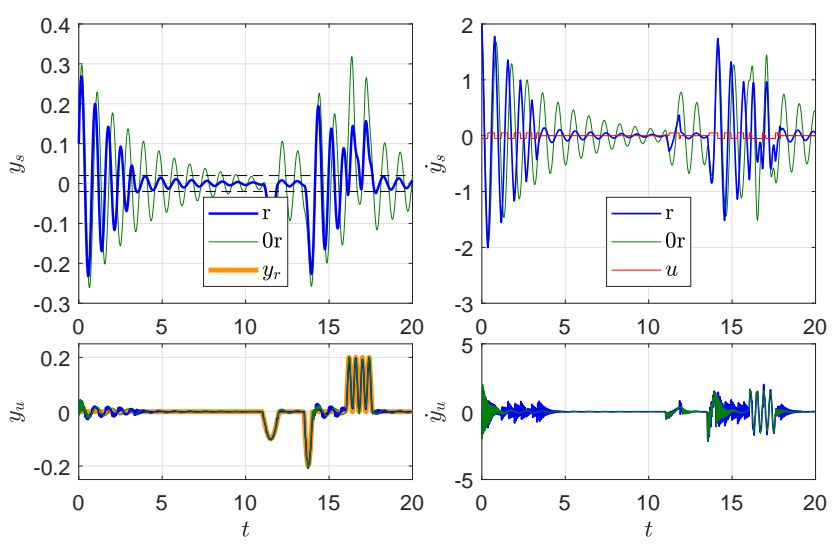

Figure 12. Time evolutions of the state variables for the actuated (blue) and passive (green) suspension. Top, left: chassis dispacement $y_{s}$. Top, right: chassis velocity $\dot{y}_{s}$. Bottom, left: tire displacement $y_{u}$. Bottom, right: tire velocity $\dot{y}_{u}$. Additionally, the input $u$ (red) and the road displacement $y_{r}$ (orange) are depicted top, right and bottom, left, respectively.

stiffness relative to the tire. For this model we have:

$$
\begin{aligned}
& m_{s} \ddot{y}_{s}+c_{s}\left(\dot{y}_{s}-\dot{y}_{u}\right)+k_{s}(\left.y_{s}-y_{u}-u\right)=0 \\
& m_{u} \ddot{y}_{u}-c_{s}\left(\dot{y}_{s}-\dot{y}_{u}\right)-k_{s}\left(y_{s}-y_{u}-u\right) \\
&+k_{t}\left(y_{u}-y_{r}\right)=0,
\end{aligned}
$$

where we consider a nonzero road displacement $y_{r}$ as the bottom, left orange trace in Figure 12, representing possible road bumps in its second half. The input $u$ is reset based only on the displacement $y_{s}$ as in (34) with only one degree of freedom, but when $u=u_{0}$ and the road excitation induces large displacements of $y_{s}$, we allow $u$ to intervene again and be accordingly reset to $u_{1}$ or $u_{2}$. With the additional parameters $m_{u}=50 \mathrm{~kg}$ and $k_{t}=250 \cdot 10^{3} \mathrm{~N} / \mathrm{m}$, we show at the top of Figure 12 the improvement of the reset law in damping oscillations and achieving a faster convergence of the chassis coordinate $y_{s}$ to its equilibrium (we also report at the bottom the unsprung-mass coordinate $y_{u}$ that follows closely the road displacement $y_{r}$ ). The good performance of the reset law with two degrees of freedom and road disturbance rests essentially upon a timescale separation between the natural frequency of the tire $\sqrt{\frac{k_{t}}{m_{u}}}=70.7 \mathrm{rad} / \mathrm{s}$ and the natural frequency of the chassis $\sqrt{\frac{k_{s}}{m_{s}}}=7.07 \mathrm{rad} / \mathrm{s}$ as in [25, Example 11.4].

\section{Conclusions and future work}

In this paper we addressed the application of relay-based reset control systems to minimal order mechanical systems. The exposition of the main results has been kept at an intuitive level, but the proofs of our main results exploit the formalism of nonlinear hybrid dynamical systems and their Lyapunov theory. Our main results apply to the two sides of the same coin, corresponding to 
sustaining and damping oscillations. Their use has been illustrated in a few simple yet relevant application cases corresponding to a hopping robot and a semi-active suspension.

The generality of the proposed hybrid approach to stability analysis calls for extensions of our methods to controlsystems design for more general mechanical systems. In particular, the minimal order modeling paradigm could be exploited together with some timescale separation (e.g., as in $[38,47]$ and references therein) to study control of hybrid periodic orbits in the context of mechatronic systems and robotic applications.

\section{References}

[1] K. J. Åström. Oscillations in systems with relay feedback. In Adaptive Control, Filtering, and Signal Processing, pages 1-25. Springer, 1995.

[2] D. P. Atherton. Early developments in nonlinear control. IEEE Control Systems, 16(3):34-43, 1996.

[3] A. Baños and A. Barreiro. Reset control systems. Springer Science \& Business Media, 2011.

[4] A. Baños, S. Dormido, and A. Barreiro. Limit cycles analysis of reset control systems with reset band. Nonlinear Analysis: Hybrid Systems, 5(2):163-173, 2011.

[5] A. Barreiro, A. Baños, S. Dormido, and J. A. GonzálezPrieto. Reset control systems with reset band: Wellposedness, limit cycles and stability analysis. Systems 86 Control Letters, 63:1-11, 2014.

[6] Z. Batts, J. Kim, and K. Yamane. Design of a hopping mechanism using a voice coil actuator: Linear elastic actuator in parallel (LEAP). In International Conference on Robotics and Automation, pages 655-660. IEEE, 2016.

[7] O. Beker, C. V. Hollot, and Y. Chait. Plant with integrator: an example of reset control overcoming limitations of linear feedback. IEEE Transactions on Automatic Control, 46(11):1797-1799, 2001.

[8] O. Beker, C. V. Hollot, Y. Chait, and H. Han. Fundamental properties of reset control systems. Automatica, 40(6):905915, 2004.

[9] J. Berner, T. Hägglund, and K. J. Åström. Asymmetric relay autotuning - Practical features for industrial use. Control Engineering Practice, 54:231-245, 2016.

[10] A. Bisoffi, F. Forni, M. Da Lio, and L. Zaccarian. Global results on reset-induced periodic trajectories of planar systems. In 2016 European Control Conference. IEEE, 2016.

[11] Y. Chait and C. V. Hollot. On Horowitz's contributions to reset control. International Journal of Robust and Nonlinear Control, 12(4):335-355, 2002.

[12] J. C. Clegg. A nonlinear integrator for servomechanisms. Transactions of the American Institute of Electrical Engineers, Part II: Applications and Industry, 77(1):41-42, 1958.

[13] F. Fichera, C. Prieur, S. Tarbouriech, and L. Zaccarian. LMIbased reset $\mathcal{H}_{\infty}$ design for linear continuous-time plants. IEEE Transactions on Automatic Control, 61(12):4157-4163, 2016.

[14] A. F. Filippov. Differential equations with discontinuous righthand sides: control systems. Kluwer Academic Publishers, 1988.
[15] R. J. Full and D. E. Koditschek. Templates and anchors: neuromechanical hypotheses of legged locomotion on land. Journal of Experimental Biology, 202(23):3325-3332, 1999.

[16] R. M. Ghigliazza, R. Altendorfer, P. Holmes, and D. Koditschek. A simply stabilized running model. SIAM review, 47(3):519-549, 2005.

[17] R. Goebel, R. G. Sanfelice, and A. R. Teel. Hybrid dynamical systems. IEEE Control Systems, 29(2):28-93, 2009.

[18] R. Goebel, R. G. Sanfelice, and A. R. Teel. Hybrid Dynamical Systems: modeling, stability, and robustness. Princeton University Press, 2012.

[19] J. W. Grizzle, C. Chevallereau, R. W. Sinnet, and A. D. Ames. Models, feedback control, and open problems of 3D bipedal robotic walking. Automatica, 50(8):1955-1988, 2014.

[20] B. Hamel. Étude mathématique des systèmes à plusieurs degres de liberte décrits par des équations linéaires avec un terme de commande discontinu. Proceedings of the Journées d'Ètudes des Vibrations AERA, 1950.

[21] M. W. Hirsch and S. Smale. Differential Equations, Dynamical Systems, and Linear Algebra (Pure and Applied Mathematics, Vol. 60). Academic Press, 1974.

[22] C. Ho, Z. Lang, and S. A. Billings. The benefits of nonlinear cubic viscous damping on the force transmissibility of a Duffing-type vibration isolator. In 2012 UKACC International Conference on Control, pages 479-484. IEEE, 2012.

[23] P. Holmes, R. J. Full, D. Koditschek, and J. Guckenheimer. The dynamics of legged locomotion: Models, analyses, and challenges. SIAM Review, 48(2):207-304, 2006.

[24] I. Horowitz and P. Rosenbaum. Non-linear design for cost of feedback reduction in systems with large parameter uncertainty. International Journal of Control, 21(6):977$1001,1975$.

[25] H. K. Khalil. Nonlinear Systems. Pearson Education, 3rd edition, 2002.

[26] D. Lakatos and A. Albu-Schäffer. Switching based limit cycle control for compliantly actuated second-order systems. In Proceedings of the 19th IFAC World Congress, pages 63926399, 2014.

[27] D. Lakatos, D. Seidel, W. Friedl, and A. Albu-Schäffer. Targeted jumping of compliantly actuated hoppers based on discrete planning and switching control. In IEEE/RSJ International Conference on Intelligent Robots and Systems, pages 5802-5808, 2015.

[28] D. Leach, F. Gunther, N. Maheshwari, and F. Iida. Linear multimodal actuation through discrete coupling. IEEE/ASME Transactions on Mechatronics, 19(3):827-839, 2014.

[29] W. S. Levine. The Control Handbook: Control System Fundamentals. CRC press, 2010.

[30] X. Lou, Y. Li, and R. G. Sanfelice. On robust stability of limit cycles for hybrid systems with multiple jumps. In Proceedings of the 5th IFAC Conference on Analysis and Design of Hybrid Systems, 2015.

[31] X. Lou, Y. Li, and R. G. Sanfelice. Results on stability and robustness of hybrid limit cycles for a class of hybrid systems. In 54th IEEE Conference on Decision and Control, pages 2235-2240. IEEE, 2015.

[32] X. Lou, Y. Li, and R. G. Sanfelice. Existence of hybrid limit cycles and Zhukovskii stability in hybrid systems. In American Control Conference, pages 1187-1192. IEEE, 2017. 
[33] G. Montaseri, M. Javad Yazdanpanah, A. Pikovsky, and M. Rosenblum. Synchrony suppression in ensembles of coupled oscillators via adaptive vanishing feedback. Chaos: An Interdisciplinary Journal of Nonlinear Science, 23(3):033122, 2013.

[34] Z. K. Peng, G. Meng, Z. Q. Lang, W. M. Zhang, and F. L. Chu. Study of the effects of cubic nonlinear damping on vibration isolations using harmonic balance method. International Journal of Non-Linear Mechanics, 47(10):1073 - 1080, 2012. Dynamics, Vibration and Control.

[35] C. Poussot-Vassal, C. Spelta, O. Sename, S. M. Savaresi, and L. Dugard. Survey and performance evaluation on some automotive semi-active suspension control methods: A comparative study on a single-corner model. Annual Reviews in Control, 36(1):148-160, 2012.

[36] M. H. Raibert. Legged robots that balance. MIT press, 1986.

[37] M. Reis and F. Iida. An energy-efficient hopping robot based on free vibration of a curved beam. IEEE/ASME Transactions on Mechatronics, 19(1):300-311, 2014.

[38] R. G. Sanfelice and A. R. Teel. On singular perturbations due to fast actuators in hybrid control systems. Automatica, 47(4):692-701, 2011.

[39] R. G. Sanfelice, A. R. Teel, and R. Sepulchre. A hybrid systems approach to trajectory tracking control for juggling systems. In 46th IEEE Conference on Decision and Control, pages 5282-5287, 2007.

[40] A. Satoh. Synthesis of output feedback linear reset control based on common quadratic Lyapunov-like function. In European Control Conference, pages 2162-2167. IEEE, 2015.

[41] S. M. Savaresi, C. Poussot-Vassal, C. Spelta, O. Sename, and L. Dugard. Semi-active suspension control design for vehicles. Elsevier, 2010.

[42] A. Sayyad, B. Seth, and P. Seshu. Single-legged hopping robotics research-A review. Robotica, 25(5):587-613, 2007.

[43] A. R. Teel, R. Goebel, B. Morris, A. D. Ames, and J. W. Grizzle. A stabilization result with application to bipedal locomotion. In IEEE 52nd Conference on Decision and Control, pages 2030-2035, 2013.

[44] Ya. Z. Tsypkin. Relay control systems. Cambridge University Press, Cambridge, England, 1984. English translation by C. Constada of the 1974 Russian edition.

[45] Ya. Z. Tsypkin and R. Herschel. Theorie der Relaissysteme der automatischen Regelung. R. Oldenbourg München, 1958.

[46] B. Vanderborght, A. Albu-Schäffer, A. Bicchi, E. Burdet, D. G. Caldwell, R. Carloni, M. Catalano, O. Eiberger, W. Friedl, G. Ganesh, et al. Variable impedance actuators: A review. Robotics and Autonomous Systems, 61(12):16011614, 2013

[47] W. Wang, A. R. Teel, and D. Nešić. Analysis for a class of singularly perturbed hybrid systems via averaging. Automatica, 48(6):1057-1068, 2012.

[48] X. Yu and F. Iida. Minimalistic models of an energy-efficient vertical-hopping robot. IEEE Transactions on Industrial Electronics, 61(2):1053-1062, 2014. 